\title{
LE SYSTÈME DE CLASSES NOMINALES DU MINYANKA
}

\author{
Sékou Coulibaly \\ Inalco \\ secoul01@gmail.com
}

Résumé : Cet article décrit le système de classes nominales du dialecte minyanka (langue sénoufo, gur) parlé dans le village de Pénesso (région de Ségou, sud-est du Mali). Il décrit les marqueurs nominaux de classe et les classes d'accord dans ce parler. Une comparaison avec les autres parlers minyanka déjà décrits montre que ce parler a subi une réduction du nombre de ses classes d'accord.

Mots-clés : minyanka, sénoufo, gur, niger-congo, classes nominales

\section{MINYANKA NOUN CLASS SYSTEM}

\author{
Sékou Coulibaly \\ Inalco \\ secoul01@gmail.com
}

\begin{abstract}
This paper is a description of the noun class system of the Minyanka dialect (Senufo, Gur) spoken in the Penesso village (Segou region, southeastern Mali). It describes the noun class markers and the agreement classes of this dialect. A comparison with other Minyanka dialects already described displays that the dialect spoken in Penesso has undergone a reduction of its agreement classes.
\end{abstract}

Key words: Minyanka, Senufo, Gur, Niger-Congo, noun classes 


\section{Introduction}

Le minyanka (ISO 639-3 myk) est une langue sénoufo du groupe gur (phylum : Niger-Congo). Cette langue a au minimum six dialectes, lesquels sont essentiellement parlés dans le sud-est du Mali dans les régions de Ségou et Sikasso. Dombrowsky-Hahn (1999; 2007) affirme qu'il est parlé également dans quelques villages situés sur le territoire burkinabé. D'après le site Ethnologue (Eberhard et al. 2021), le nombre des locuteurs du minyanka s'élevait à 738.000 en 2000. Le dialecte qui fait l'objet de cet article est parlé dans le village de Pénesso, situé à une cinquantaine de kilomètres à l'est de la ville de Bla (région de Ségou), dans la commune rurale de Yangasso. Selon les chiffres du recensement général de la population malienne, le village de Pénesso comptait 2.344 habitants en $2015: 1.149$ hommes et 1.195 femmes. Les données ayant servi à l'élaboration de cet article ont été collectées à Pénesso entre février et mars 2017, dans le cadre de mon travail de terrain pour ma thèse de doctorat. En plus de cela, je suis moi-même locuteur natif de cette langue.

La méthodologie adoptée ici s'inspire largement du modèle présenté par des guristes dans Miehe \& Winkelmann (2007) et d'une certaine mesure de Güldemann \& Fiedler (2019). Mais à la différence des auteurs se trouvant dans Miehe \& Winkelmann (2007), qui utilisent une numérotation des classes d'après une reconstruction des classes du proto-gur, je n'utiliserai pas de numéros pour désigner les classes d'accord du minyanka pour la simple raison que celles-ci se présentent de façon différente dans le parler Pénesso. Par souci de clarté, je désignerai les classes d'accord par les consonnes qui les caractérisent. En effet, en sénoufo, chaque classe d'accord est caractérisée par une consonne qui intervient dans tous les mécanismes d'accord (pronoms et dépendants du nom), où elle est suivie d'une voyelle qui indique la fonction grammaticale. Ce propos de Carlson (1994: 75) à propos du supyiré résume bien la situation :

In general, one can say that the noun class is marked by a consonant, and that other functions are marked by the following vowel. It is thus 
possible to speak of "class consonants": each noun class has a typical consonant or family of consonants (sharing the same point of articulation) which recur again and again in the morphological marking of that class.

Dans cet article, je parlerai d'abord du système nominal de classes $(\S 2)$, ensuite des formes nominales et des genres $(\S 3)$ et enfin des mots soumis à l'accord de classe $(\S 4)$.

\section{Système nominal de classes}

La morphologie des noms en minyanka est caractérisée par la présence obligatoire de suffixes qui, d'une part, marquent l'opposition de nombre (singulier vs. pluriel), et d'autre part sont conditionnés par l'appartenance du nom à un genre (sous-ensemble de lexèmes nominaux qui ont les mêmes propriétés en tant que contrôleurs d'un mécanisme d'accord ayant pour cibles leurs modifieurs ou les pronoms dont ils sont les antécédents). En d'autres termes, le suffixe flexionnel que comporte chaque forme nominale, outre sa fonction de marque de nombre, marque l'appartenance de la forme nominale à une classe d'accord manifestée par le choix des marques d'accord que portent ses modifieurs, ou les pronoms dont elle est l'antécédent.

On distingue 8 marqueurs nominaux de classe en minyanka, lesquels sont suffixés à la base, et marquent l'opposition du nombre. Ils se répartissent de la façon suivante : 3 paires de suffixes marquant l'opposition singulier vs. pluriel s'adjoignant à des noms comptables, et 2 suffixes s'adjoignant à des noms non-comptables. Dans la Figure 1, les suffixes nominaux sont précédés d'un tiret et les pronoms de classe (ou pronoms anaphoriques) sont marqués en gras. Les lignes reliant les différents pronoms marquent les appariements singulier/pluriel. Les pronoms tí et $k i$, précédés respectivement des suffixes $-r V$ et $-m V$, représentent les classes des noms non-comptables. Les lettres en majuscule se trouvant aux deux extrémités de la Figure 1 représentent les classes d'accord des différentes formes nominales du minyanka de Pénesso. 


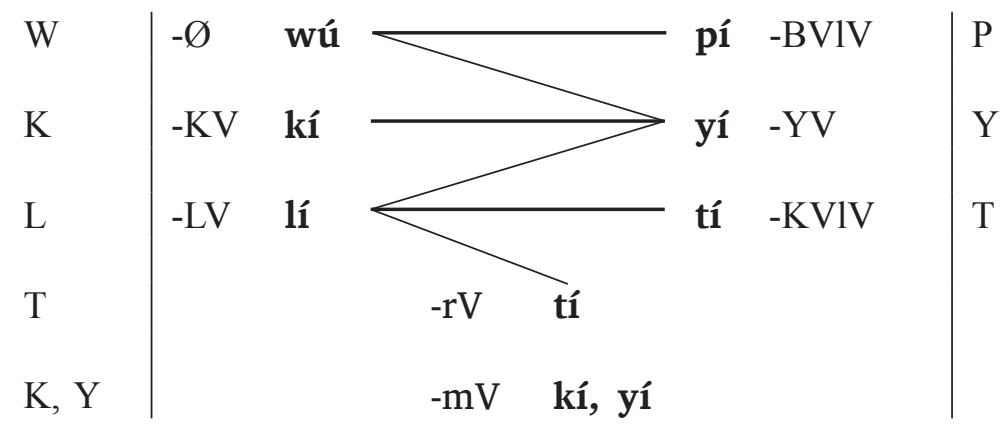

Figure 1. Vue d'ensemble des suffixes nominaux de l'indéfini et des pronoms de classe.

Nous constatons que dans la Figure 1 certains pronoms de classe apparaissent deux fois à des endroits différents. Par exemple, le pronom de classe tí représente à la fois les noms qui prennent le suffixe de pluriel -KVlV (ex. wyèkèlè '(petits) trous') et ceux qui prennent le suffixe $-r V$ comprenant des noms non-comptables (hárá 'viande') et certains noms au pluriel à valeur diminutive (ex. nj̀ Yう̀pìré 'enfants'). En d'autres termes, les suffixes $-K V l V$ et $-r V$ ont en commun le fait de s'adjoindre à des noms désignant de petites choses.

Le pronom de classe kí représente à la fois les noms qui portent le suffixe de singulier $-K V$ et des noms non-comptables portant le suffixe $-m V$. Les exemples suivants montrent que les noms à suffixe $-K V l V$ et les noms à suffixe $-r V$ ont les mêmes propriétés d'accord (1)-(3). Les exemples (4)-(5) montrent que les noms portant le suffixe de singulier $-K V$ et ceux portant le suffixe $-m V$ ont les mêmes propriétés d'accord.
(1) a. Wyè-kèlè
wà

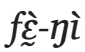
nà.
trou-PL.INDF
être.PRS.AFF
habit-SG.DEF
sur
'L'habit est troué. (litt. Les trous sont sur l'habit.)'
b. Tí wá fî̀-
3PL.T être.PRS.AFF habit-SG.DEF sur
'(litt.) Ils (les trous) sont sur 1'habit.'




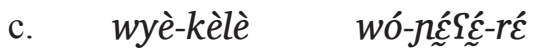
trou-PL.INDF ADJ-être.nombreux-T
'beaucoup de trous'

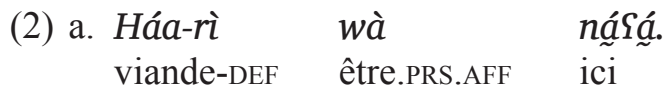
'La viande est là.'
b. Tí wá náạáa
3SG.T être.PRS.AFF ici
'Elle (la viande) est là.'

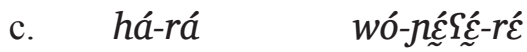
viande-INDF ADJ-être.nombreux-T
'beaucoup de viande'

(3) a. Nò̀Ỳ̀-pǔi-rì

wà

nåạá.

personne-enfant-DEF être.PRS.AFF ici

'Les enfants sont là.'

b. Tí wá nạ́ạá.

3PL.T être.PRS.AFF ici

'Ils (les enfants) sont là.'

c. nว̀ेญ̀-pì-ré

personne-enfant-INDF

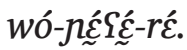

'Beaucoup d'enfants.'

(4) a.

$\begin{array}{lll}\text { Cí-kí } & \text { wá } & \text { tò. } \\ \text { arbre-SG.DEF } & \text { PRF.AFF } & \text { tomber }\end{array}$

'L'arbre est tombé.'

b. Ki wá tò.

3SG.K PRF.AFF tomber

'Il (l'arbre) est tombé.'

c. cí-tớñó-kó

arbre-être.grand-SG.INDF

'un grand arbre'

(5) a. $\int \grave{\imath}-m i$

wà tăa.

bière.de.mil-DEF PRF.AFF être.agréable

'La bière de mil est agréable.' 


\section{b. Kí wá tăăa. \\ 3SG.K PRF.AFF être.agréable \\ 'Elle (la bière de mil) est agréable.'

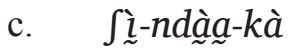 \\ bière.de.mil-être.agréable-INDF \\ 'une bonne bière de mil'}

Dans ma liste lexicale, jómí 'la parole' est le seul nom à suffixe $-m V$ à s'accorder en classe $\mathrm{Y}$, laquelle est la classe des noms au pluriel à suffixe $-Y V$. Cela s'explique par le fait que jómí est sémantiquement perçu en minyanka comme un nom au pluriel dont la traduction exacte est 'les paroles'. Les exemples (6)-(7) montrent que jómí et les noms à suffixe $-Y V$ gouvernent les mêmes accords.

(6)

a. Má jó-mì wà jèç⿳亠丷厂.

2SG parole-DEF PRF.AFF être.nombreux

'Tu parles trop. (litt. Tes paroles sont nombreuses.)'

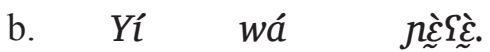

3PL.Y PRF.AFF être.nombreux

(litt.) 'Elles (tes paroles) sont nombreuses.'

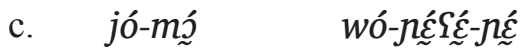

parole-INDF ADJ-être.nombreux-Y

'beaucoup de paroles'

(7) a. Mè

$f_{\mathcal{E}}^{\prime}-n \grave{i}$

wà

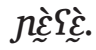

1SG.EMPH habit-PL.DEF PRF.AFF être.nombreux

'Mes habits sont nombreux.'

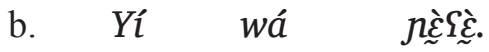
3PL.Y PRF.AFF être.nombreux
'Ils (mes habits) sont nombreux.'

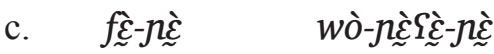
habit-INDF ADJ-être.nombreux-Y
'beaucoup d'habits'


Comme nous pouvons le constater dans les exemples ci-dessus, les noms à suffixe $-K V l V$ et ceux à suffixe $-r V$ gouvernent les mêmes accords, les noms à suffixe $-K V$ et ceux à suffixe $-m V$ aussi gouvernent les mêmes accords. Les noms à suffixe $-Y V$ et le nom jómí 'les paroles' aussi ont les mêmes accords.

Il convient de souligner que dans ma liste lexicale, il y a deux noms portant le suffixe de pluriel $-K V l V$, qui s'accordent toujours en classe $\mathrm{K}$ pluriel et non en classe $\mathrm{T}$; il s'agit de njèkèlè 'balafon' et jàsàlà 'haricot'. Mais ces noms ont la particularité de ne pas avoir de forme de singulier; ils s'emploient toujours au pluriel. Il convient de souligner également que lors de la collecte de mes données, j'ai remarqué que certains locuteurs (une minorité) du minyanka de Pénesso accordaient les noms à suffixe $-m V$ en classe $\mathrm{P}$ et acceptaient également l'accord en classe K pour ces mêmes noms. Ces irrégularités montrent à suffisance que le processus de réduction du nombre des classes d'accord n'est pas encore terminé à Pénesso.

Si l'on se réfère à ce qui vient d'être dit, on peut dire que les noms à suffixe $-K V l V$ et ceux à suffixe $-r V$ appartiennent à la même classe d'accord : la classe $\mathrm{T}$. Les noms au singulier à suffixe $-K V$ et ceux à suffixe - $m V$, excepté jómí 'les paroles' appartiennent à la même classe d'accord : la classe $\mathrm{K}$. Les noms au pluriel à suffixe $-Y V$ et le nom jómí appartiennent à la même classe d'accord : la classe Y. A partir de là, on peut conclure qu'il y a 8 suffixes nominaux de classe et seulement 6 classes d'accord en minyanka de Pénesso, lesquelles sont : classe W, classe P, classe $\mathrm{K}$, classe $\mathrm{Y}$, classe L et classe T (voir Figure 2).

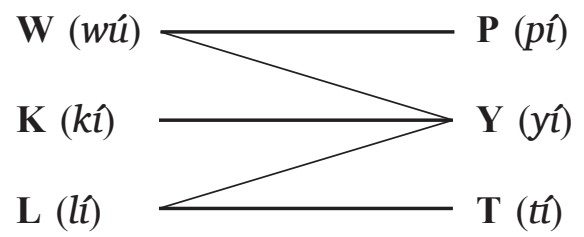

Figure 2. Classes d'accord 


\section{Formes nominales et genres}

\subsection{Suffixes nominaux de classe}

Il y a deux types de suffixes nominaux en minyanka : ceux de l'indéfini et ceux du défini. Nous parlerons d'abord des premiers, ensuite nous parlerons des seconds en \$3.2. La structure des suffixes nominaux de l'indéfini est $\mathrm{CV}(\mathrm{CV})$, et il y a une assimilation entre la dernière voyelle de la base nominale et la ou les voyelles du suffixe. En effet, dans un nom simple du minyanka, toutes les voyelles seront soit antérieures soit postérieures. Lorsque la dernière voyelle de la base est fermée, la ou les voyelles du suffixe seront plus ouvertes d'un ou

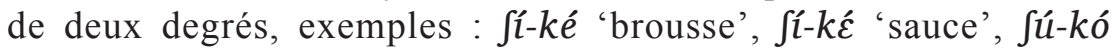
'mortier', fú-kón 'cheval'.

Lorsque la dernière voyelle de la base est mi-fermée, celle-ci est reproduite au suffixe, sauf si la consonne initiale du suffixe est une nasale, auquel cas la voyelle du suffixe est toujours mi-ouverte,

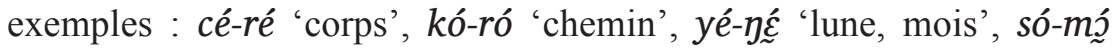
'sel'.

Lorsque la dernière voyelle de la base est mi-ouverte ou ouverte, elle est exactement reproduite au suffixe, exemples : $w \hat{\varepsilon}-r \varepsilon$ 'remède', nó-kó 'plaie', há-rá 'viande', cálá-ká 'gouttière'.

Il faut noter que lorsque la consonne initiale du suffixe est une nasale, la voyelle qui lui succède est toujours mi-ouverte ; elle est ouverte seulement si la dernière voyelle de la base est ouverte,

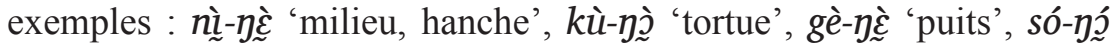

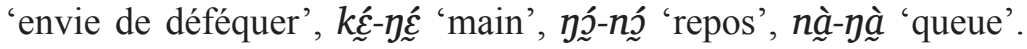

Ces règles sont reprises dans le Tableau 1.

${ }^{1}$ Lorsque la dernière voyelle de la base est $u$, la voyelle du suffixe est toujours $o$ si la consonne initiale du suffixe est orale, sauf pour le nom fú-kó 'cheval'. 
Tableau 1

Voyelles de la base vs. voyelles du suffixe à la forme indéfinie des noms

\begin{tabular}{|c|c|c|c|c|c|}
\hline \multicolumn{2}{|c|}{ voyelles antérieures } & \multicolumn{2}{|c|}{ voyelle centrale } & \multicolumn{2}{|c|}{ voyelles postérieures } \\
\hline base & suffixe & base & suffixe & base & suffixe \\
\hline $\mathrm{i}$ & e, $\varepsilon$ & & & $\mathrm{u}$ & o, o \\
\hline $\mathrm{e}$ & $\mathrm{e}, \varepsilon$ & & & o & o, o \\
\hline$\varepsilon$ & $\varepsilon$ & a & $\mathrm{a}$ & o & o \\
\hline
\end{tabular}

\subsection{Les genres et leurs contenus sémantiques}

En minyanka de Pénesso, la forme de citation des noms est le défini, dont le marqueur se superpose généralement au suffixe de l'indéfini, mais dans certains cas, les suffixes du défini se substituent à ceux de l'indéfini. Les suffixes du défini des noms sont présentés dans le Tableau 2 ci-dessous.

\section{Tableau 2}

Suffixes du défini des noms

\begin{tabular}{|ccc|}
\hline Singulier & non-comptable & Pluriel \\
\hline$-u$ & & $-p i$ \\
$-k i$ & & $-y i$ \\
$-l i$ & & $-k i$ \\
& & \\
& $-r i$ & \\
& $-m i$ & \\
\end{tabular}

Dans les sous-sections qui suivent, nous présenterons d'abord les noms à leurs formes indéfinies (singulier/pluriel), et à la fin de chaque sous-section, nous donnerons les quatre formes des noms côte à côte (indéfinie singulier, définie singulier, indéfinie pluriel, définie pluriel). 


\subsubsection{Genre W/P}

Tous les noms propres de personne s'accordent en classe W. C'est dans ce genre que l'on trouve la plupart des noms d'humains et tous les noms désignant les membres des ethnies. On y rencontre également les noms d'êtres surnaturels, de certains animaux, et de certains inanimés. Les suffixes nominaux ainsi que les pronoms de classe du genre W/P sont présentés dans le Tableau 3 ci-dessous.

Tableau 3

Suffixes nominaux et pronoms de classe du genre W/P

\begin{tabular}{|l|l|l|}
\hline & Singulier & Pluriel \\
\hline $\begin{array}{l}\text { suffixes de } \\
\text { l'indéfini }\end{array}$ & $-\emptyset$, & $-l V /-n V$, \\
$-V$ & $\begin{array}{l}-b V l V /-m V n V,-m V V,-m V, \\
-k V l V /-\eta V n V,-l V /-n V, \\
-V l V /-V n V,-l V l V /-n V n V,-V^{2}\end{array}$ \\
\hline $\begin{array}{l}\text { suffixes du } \\
\text { défini }\end{array}$ & $-u$ & $-p i$ \\
\hline $\begin{array}{l}\text { pronoms de } \\
\text { classe }\end{array}$ & $w u ́$ & $p i ́$ \\
\hline
\end{tabular}

$-\emptyset /-b V I V$

ć́ / cèbèlè 'femme'

$-\varnothing /-m V n V$

En contexte nasal, $-b V l V$ se réalise $-m V n V$.

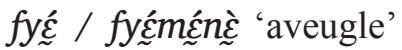

sàYàzì / sàYàzìmźnì̃ 'chat'

${ }^{2}$ Le suffixe de pluriel $-b V l V$ et ses allomorphes ont en réalité une valeur neutre. Nous les appelons suffixes de l'indéfini pluriel pour les différencier de -pi qui a toujours la valeur du défini. 


\section{$-\emptyset /-m V V$}

L'allomorphe $-m V V$ peut être analysé comme issu de $-m V n V$ dont le $n$ intervocalique s'est estompé.

nà / nàmàa 'hommes'

\section{$-\emptyset /-V I V$}

L'allomorphe $-V l V$ peut être analysé comme issu de $-b V l V$ dont la consonne initiale s'est élidée.

fyè / fyèelè 'python'

já / jáalà 'fils de'

\section{$-\emptyset /-V n V$}

L'allomorphe $-V n V$ est la variante nasale de $-V l V$.

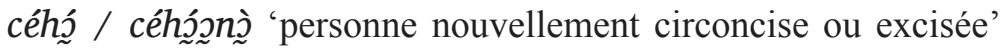
pà / pàanà 'varan d'eau'

\section{$-\emptyset /-l V$}

L'allomorphe $-l V$ peut être considéré comme la forme tronquée de $-b V l V$.

métírí / métírilè 'maître'

póró / pórólò 'fille de'

\section{$-\varnothing /-n V$}

L'allomorphe $-n V$ est la variante de $-l V$ en contexte nasal.

fúfùmó / Júfùmónò 'mouche'

tùndù̀y / tùndù̀ỳ̀ñ 'messager'

\section{$-\varnothing /-V$}

On peut expliquer l'allomorphe $-V$ en postulant la chute de la consonne $l$ de la forme $-l V$.

fyá / fyàa 'poisson'

$\int \hat{\varepsilon} / \int_{\mathcal{E} \mathcal{E}}$ 'personne' 
$-\varnothing /-k V l V$

tứndú / túndùkòlòs 'forgeron'

gó / hùkólò 'défunt'

\section{$-\eta V n V$}

Cet allomorphe est la variante de $-k V l V$ en contexte nasal. Dans

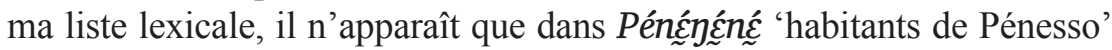

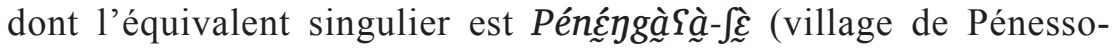
personne) 'ressortissant de Pénesso'.

\section{$-V /-l V l V$}

Dans ma liste lexicale, seuls deux exemples ont été trouvés avec l'allomorphe -lVlV.

pyà / pìlèlè pìlèe 'enfant'

tùpyà / tùpilélè 'personne'

$-l V /-V$

நgòlò / hòo 'poulet'

Ici, on a affaire à une alternance de la consonne initiale $\eta g / h$.

$-n V /-V$

L'allomorphe $-n V$ est sans doute la variante de $-l V$ en contexte nasal.

nằṅ̀̀n

\section{$-n V /-m V$}

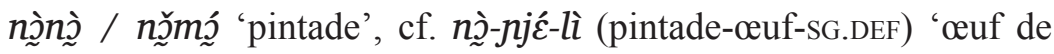
pintade'

${ }^{3}$ La dernière voyelle de la base se dénasalise au pluriel.

${ }^{4}$ Il est curieux de constater que la forme de singulier du nom gó 'défunt' est homonyme du verbe gó 'tuer' et que la base du pluriel hù- ressemble au verbe hùû 'mourir'. Ici, on peut parler d'allomorphie supplétive de la base. 
Le suffixe du défni singulier - $u$ n'apparaît sous cette forme que dans de rares cas tels que la forme nominalisée des verbes se terminant par la voyelle $i$, exemples : nìrì-u 'le fait de se lever', cf. yìrî 'se lever', vì-ù 'le fait de germer', cf. fî̀ 'germer', etc. Dans les autres cas, il se réalise :

- $u$ en fusionnant avec un nom se terminant par $i$,

- $o$ en fusionnant avec un nom se terminant par $e$,

- $\supset$ en fusionnant avec un nom se terminant par $\varepsilon$ ou $a$.

Quant au suffixe du défini pluriel -pi, il se superpose au suffixe de l'indéfini pluriel, voir Tableau 4.

Tableau 4

Formes de singulier vs. formes de pluriel des noms du genre W/P

\begin{tabular}{|c|c|c|c|c|}
\hline SG.INDF & SG.DEF & PL.INDF & PL.DEF & Signification \\
\hline sàYàzì & sàYàzù & sàYàzìmźćñ & 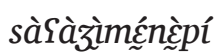 & 'chat' \\
\hline$c \hat{\varepsilon}$ & $c \hat{\jmath}$ & cદ̀bèlè & cદ̇bšlèpì & 'femme' \\
\hline$n \grave{a}$ & nџ̀ & nà̃màa & nàmmàapì & 'homme' \\
\hline
\end{tabular}

\subsubsection{Genre $\mathrm{W} / \mathrm{Y}$}

Les noms appartenant à ce genre sont majoritairement des emprunts. On y trouve des noms d'animaux, des noms d'inanimés et des noms abstraits. Les suffixes nominaux et les pronoms de classe du genre W/Y sont présentés dans le Tableau 5.

Tableau 5

Suffixes nominaux et pronoms de classe du genre $\mathrm{W} / \mathrm{Y}$

\begin{tabular}{|l|l|l|}
\hline & Singulier & Pluriel \\
\hline suffixes de l'indéfini & $-\emptyset$ & $-y V /-n V$ \\
\hline suffixes du défini & $-u$ & $-y i /-n i$ \\
\hline pronoms de classe & $w u ́$ & $y i$ \\
\hline
\end{tabular}


$-\emptyset /-y V$

ségá / sékàyà 'caprin'

búkúlú / búkúlúyó 'boucle d'oreille'

$-\varnothing /-n V$

Lallomorphe $-n V$ est la variante de $-y V$ en contexte nasal.

bànà / bànànàa 'maladie'

jàmù / jàmùñ̀̃ 'nom de famille'

Quelques exemples des noms du genre W/Y sont donnés dans le Tableau 6.

Tableau 6

Formes de singulier vs. formes de pluriel des noms du genre W/Y

\begin{tabular}{|c|c|c|c|c|}
\hline SG.INDF & SG.DEF & PL.INDF & PL.DEF & Signification \\
\hline ségá & séĝे & sékàyà & sékàyì & chèvre \\
\hline búkúlú & búkúlû & búkúlúyó & búkúlúyí & boucle d'oreille \\
\hline bànà & bàñ̀ & bànàngà & bànànì & maladie \\
\hline jàmừ & jàmûu & jàmù̀ñ̀ & jàmùnì & nom de famille \\
\hline
\end{tabular}

\subsubsection{Genre $\mathrm{K} / \mathrm{Y}$}

La majorité des noms appartenant à ce genre sont ceux d'inanimés, souvent de grande taille. On y trouve des noms d'arbres, de substances liquides, d'animaux, d'oiseaux et de certaines parties du corps. Les suffixes nominaux et les pronoms de classe du genre $\mathrm{K} / \mathrm{Y}$ sont présentés dans le Tableau 7.

Tableau 7

Suffixes nominaux et pronoms de classe du genre $\mathbf{K} / \mathbf{Y}$

\begin{tabular}{|l|l|l|}
\hline & Singulier & Pluriel \\
\hline suffixes de l'indéfini & $-k V /-\eta V,-\emptyset$ & $-y V /-n V$ \\
\hline suffixes du défini & $-k i /-\eta i,-\varsigma V y i /-\varsigma \tilde{V} n i$ & $-y i /-n i$ \\
\hline pronoms de classe & $k i ́$ & $y i ́$ \\
\hline
\end{tabular}


$-k V /-y V$

cíké / cíyé 'arbre'

kùlókùlòkò / kùlókùlòyò 'dindon'

\section{$-\boldsymbol{\eta} \boldsymbol{V} / \boldsymbol{- n} \boldsymbol{V}$}

Les allomorphes $-\eta V /-n V$ sont les variantes de $-k V /-y V$ en contexte nasal.

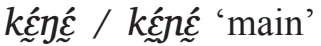

cáñá / cáñá 'jour’

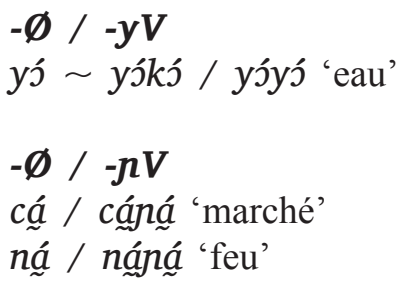

Au défini, les suffixes de l'indéfini sont remplacés par ceux du défini. Le suffixe du défini singulier - $k i$ se réalise - $\$ V y i$ lorsque la dernière voyelle de la base est $a, \mathcal{\varepsilon}$ ou 2 . Il se réalise $-\varsigma \tilde{V}$ ji lorsque la dernière voyelle de la base est $\underset{\sim}{a}, \underset{\sim}{\mathcal{E}}$ ou $\underset{\sim}{2}$. En voyant ces allomorphes, on est tenté de les analyser comme la combinaison de deux suffixes $-\varsigma V /-\varsigma \tilde{V}$ et $-y i$ / -ni qui correspondent respectivement au suffixe de singulier et de pluriel, car en minyanka, la fricative pharyngale $\subseteq$ fonctionne comme l'allophone de l'occlusive vélaire $k$ se trouvant entre deux voyelles ouvertes identiques ou entre deux voyelles mi-ouvertes identiques. Si l'on admet cette hypothèse, on s'attendrait à ce que les noms portant -SVyi / - $\$ \tilde{V}$ ni s'accordent en classe Y (classe de pluriel), or il se trouve que ceux-ci s'accordent plutôt en classe K (classe de singulier), et -SVyi et - $\Upsilon \tilde{V}$ ni sont remplacés respectivement par -yi et -ni au défini pluriel. Cela nous amène à dire que - $\$ V y i$ et $-\varsigma \tilde{V}$ ni sont des allomorphes du suffixe du défini singulier -ki. Cette hypothèse paraît plus plausible dans la mesure où les formes verbales du minyanka se terminant par la syllabe ki précédée d'une voyelle mi-ouverte ou 
ouverte sont souvent prononcées avec le son [SVyi] à la fin, exemples : kèbèkì kèbèč̀yì 'casser', kálákí káláSáyí ', détruire, endommager'. Quelques exemples des noms du genre $\mathrm{K} / \mathrm{Y}$ sont donnés dans le Tableau 8.

Tableau 8

Formes de singulier vs. formes de pluriel des noms du genre K/Y

\begin{tabular}{|c|c|c|c|c|}
\hline SG.INDF & SG.DEF & PL.INDF & PL.DEF & Signification \\
\hline cíké & cîkí & cíyé & cíyí & arbre \\
\hline$k \mathcal{E} \eta \underline{\tilde{E}}$ & kÉgì & 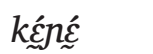 & kếnì & main, bras \\
\hline sèrèkè & sc̀rદ̀Yદ̀yì & sc̀rc̀y & sčrc̀yì & dos \\
\hline$c \stackrel{a}{a}$ & cắSání & cạ́ná & cạ́ní & marché \\
\hline kórókś & kóróৎóyí & kóróyó & kóróyí & pirogue \\
\hline
\end{tabular}

\subsubsection{Genre L/Y}

Dans ma liste lexicale, seules deux paires de singulier-pluriel appartiennent à ce genre, et ont la particularité de porter deux suffixes au pluriel : un suffixe de singulier auquel se superpose le suffixe de pluriel. Les suffixes nominaux ainsi que les pronoms de classes du genre L/Y sont présentés dans le Tableau 9.

Tableau 9

Suffixes nominaux et pronoms de classe du genre $L / Y$

\begin{tabular}{|l|l|l|}
\hline & Singulier & Pluriel \\
\hline suffixes de l'indéfini & $-l V$ & $-y V$ \\
\hline suffixes du défini & $-l i$ & $-y i$ \\
\hline pronoms de classe & $l i$ & $y i ́$ \\
\hline
\end{tabular}

$-l V /-l V-y V$

tóló / tólóyó 'pied, jambe' 


\section{$-l V /-r V-y V$}

hálá / kèrèyè 'affaire'

Les noms du genre L/Y sont présentés dans le Tableau 10.

Tableau 10

Formes de singulier vs. formes de pluriel des noms du genre L/Y

\begin{tabular}{|l|l|l|l|l|}
\hline SG.INDF & SG.DEF & PL.INDF & PL.DEF & Signification \\
\hline tóló $\sim$ tóró & tólí & tólóyó & tólóyí & pied \\
\hline hálá & hálì & kèrèyè & kèrèyì & affaire \\
\hline
\end{tabular}

\subsubsection{Genre L (-LV) / T (-KVlV)}

La plupart des noms de cet appariement désignent des choses de petite taille ou des choses de forme ronde ou ovale. Les suffixes nominaux ainsi que les pronoms de classe du genre L/T sont donnés dans le Tableau 11.

Tableau 11

Suffixes nominaux et pronoms de classe du genre $\mathrm{L}(-L V) / \mathrm{T}(-K V L V)$

\begin{tabular}{|l|l|l|}
\hline & Singulier & Pluriel \\
\hline suffixes de l'indéfini & $-l V /-n V$ & $\begin{array}{l}-k V l V /-\eta V n V^{6}, \\
-\varsigma V l V /-\varsigma V \tilde{V} n \tilde{V},-V,-V V,-V k V\end{array}$ \\
\hline suffixes du défini & $-l i /-n i$ & $-k i,-\varsigma V y i /-\varsigma \tilde{V} n i$ \\
\hline pronoms de classe & $l i$ & $\mathfrak{t}^{\prime}$ \\
\hline
\end{tabular}

$$
\begin{aligned}
& -l V \text { / - } \mathbf{k V l V} \\
& \text { célé / cèkèlè 'cuisse' } \\
& \text { wyélé / wyèkèlè 'trou' }
\end{aligned}
$$

${ }^{5}$ Allomorphie supplétive de la base.

${ }^{6}$ A l'instar du suffixe de pluriel $-b V l V$ (classe P), le suffixe de pluriel $-k V l V$ (classe T) aussi a une valeur neutre. Nous l'appelons suffixe de l'indéfini pour le différencier du suffixe de pluriel défini $-k i$. 
$-n V /-\eta \boldsymbol{n} \boldsymbol{V}$

$-n V$ et $-\eta V n V$ sont respectivement les allomorphes de $-l V$ et $-k V l V$ en contexte nasal.

péné $/$ péyćcnc̀ 'ruche'

\section{$-l V$ / - $S V l V$}

$-\varsigma V I V$ est l'allomorphe de $-k V l V$ lorsque la dernière voyelle de la base est $\varepsilon, \supset$ ou $a$.

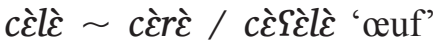

lálá / làYàlà 'grossesse'

$w^{w}$ ว́ló / $w^{w}$ ว̀५j̀lò ‘étoile’

$-n V$ / $-\boldsymbol{S} V \boldsymbol{n} V$

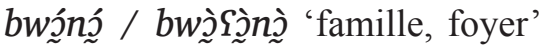

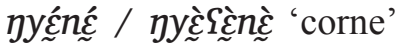

$-l V /-V$

yélé / yèc 'année'

góló / hòo 'gourde (calebasse)', alternance consonantique g/h.

$-n V /-\tilde{V}$

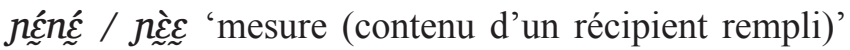

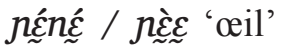

$-l V /-V V$

bílé / pyàa 'graine'

Ici, il y a une alternance de la consonne initiale $b / p$.

Le suffixe de pluriel $-V k V$ a souvent (mais pas toujours) une valeur diminutive ; il peut s'adjoindre à des bases se terminant par une voyelle orale ou nasale sans distinction du degré d'aperture de celle-ci.

\section{valeur diminutive}

$-V k V$ 
céekè 'petites cuisses'

tóokj̀ 'petits pieds'

finăakà 'garçonnets'

\section{valeur neutre}

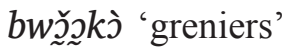

kàyěekè 'quartiers'

kóokò 'chemins, routes'

kǔukò 'villages'

Au défini singulier, le suffixe de l'indéfini $-l V /-n V$ est remplacé par -li / -ni. Au défini pluriel, le suffixe du défini -ki et ses allomorphes - $Y V y i$ / - $\{\tilde{V} n i$ se superposent au suffixe de l'indéfini pluriel, voir Tableau 12.

Tableau 12

Formes de singulier vs. formes de pluriel des noms du genre L/T

\begin{tabular}{|c|c|c|c|c|}
\hline SG.INDF & SG.DEF & INDF.PL & DEF.PL & Signification \\
\hline célé & célì & cèkèlè & cèkèlèkì & cuisse \\
\hline lálá & lálí & làYàlà & làYàlàYàyì & grossesse \\
\hline 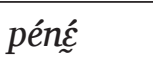 & pénì & 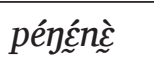 & 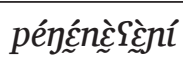 & ruche \\
\hline kùlò & kùlì & kǔukò & kǔukòkí & village \\
\hline
\end{tabular}

\subsubsection{Genre L $(-L V) / \mathrm{T}(-r V)$}

Certains noms portant le suffixe $-l V /-n V$ au singulier forment leur pluriel en prenant le suffixe $-r V$ (suffixe des noms collectifs, et de certains noms massifs). Ils ont tous une valeur diminutive et certains d'entre eux sont composés de deux bases dont la seconde est bí-lé (graine-SG.INDF) 'graine'. Au pluriel le nom bílé est remplacé par pí-ré (enfant/graine-PL.INDF) 'enfants, graines'. D'autres sont des noms simples. 
$-l V /-r V$

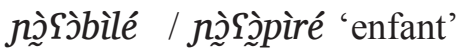

cébilé / cépíré 'jeune épouse'

ggòbìlé / ggòpìré 'poussin'

$-n V /-r V$

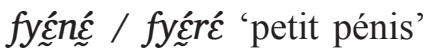

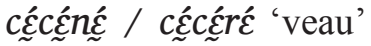

Quelques exemples des noms du genre L (-LV) / T (-rV) sont présentés dans le Tableau 13.

Tableau 13

Formes de singulier vs. formes de pluriel des noms du genre $L / T$

\begin{tabular}{|c|c|c|c|c|}
\hline SG.INDF & SG.DEF & PL.INDF & PL.DEF & Signification \\
\hline nə̀)う̄bìlé & 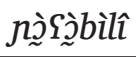 & nગેYう̀pìré & 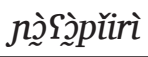 & enfant \\
\hline cébílé & cébílì & cépíré & cépíirì & jeune épouse \\
\hline fy'ָ̃́n & fy’̛̃ní & fy'śr & fyéž & petit pénis \\
\hline 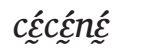 & cẼç્હ́nì & 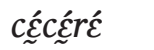 & 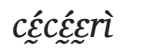 & veau \\
\hline
\end{tabular}

3.2.7. Classe $\mathrm{T}(-r V)$

Hormis des noms à suffixe $-l V /-n V$ qui forment leur pluriel en prenant le suffixe $-r V$, les noms qui portent le suffixe $-r V$ n'ont pas d'appariement singulier-pluriel. Cette classe contient des noms massifs, des noms abstraits, et des noms collectifs. Les suffixes nominaux et le pronom anaphorique de la classe T (-rV) sont donnés dans le Tableau 14.

\section{Tableau 14}

Suffixes nominaux et pronom anaphorique de la classe $T$

\begin{tabular}{|l|l|}
\hline suffixe de l'indéfini & $-r V$ \\
\hline suffixe du défini & $-r i$ \\
\hline pronom de classe & $t^{\prime}$ \\
\hline
\end{tabular}




\section{céré 'corps' \\ fíré 'excrément' \\ hárá 'viande' \\ sহ̃ró 'douleur' \\ nà̀gà̀à 'vol' \\ kànż̀̇̀̀ ' 'gravier' \\ ndìrè 'hirondelle'}

Au défini, le suffixe - $r V$ est remplacé par -ri. L'adjonction du suffixe du défini -ri entraîne parfois une longueur de la voyelle de la base. Cette longueur vocalique peut s'expliquer en disant que le suffixe du défini s'est superposé à celui de l'indéfini, et comme les deux suffixes ont la forme $r V$, le $r$ du suffixe de l'indéfini, pour des raisons phonétiques, s'est estompé, provoquant ainsi une voyelle longue.

forme indéfinie + suffixe du défini

céré + -ri > céerí 'corps'

firé $+-r i>$ fiirì 'excrément'

hárá + -ri > háarì 'viande'

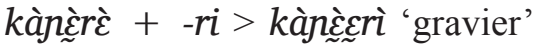

\subsubsection{Classe $\mathrm{K}(-\mathrm{mV})^{7}$}

Les noms de cette classe sont non-comptables. Cette classe est constituée d'une part de noms de substances liquides ou pulvérulentes et d'autre part de noms abstraits. Les suffixes nominaux et le pronom anaphorique de la classe K (-mV) sont présentés dans le Tableau 15.

\section{Tableau 15}

\section{Suffixes nominaux et pronom anaphorique de la classe $\mathrm{K}$}

\begin{tabular}{|l|l|}
\hline suffixe de l'indéfini & $-m V,-\emptyset$ \\
\hline suffixe du défini & $-m i$ \\
\hline pronom de classe & $k i ́$ \\
\hline
\end{tabular}

${ }^{7}$ Rappelons que certains locuteurs accordent les noms à suffixe $-m V$ en classe $\mathrm{P}$. 
$-m V$

júrímé 'lait'

fírímé 'urine'

sómó 'sel'

kácámá 'fatigue'

fwòmว̀ 'décès'

f'́rémé 'aisance’

$-\varnothing$

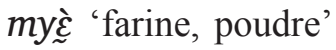

Au défini, le suffixe de l'indéfini est remplacé par -mi.

jírímí 'le lait'

fírími 'l'urine'

sómì 'sel'

myغ̇̀mì 'la farine, poudre'

\subsubsection{Classe $\mathrm{Y}(-m V)$}

Jómð́ 'paroles' est le seul nom à suffixe $-m V$ à s'accorder en classe Y. A la forme définie, le suffixe de l'indéfini - $m V$ est remplacé par - $m i$; ce qui donne jómí 'les paroles'. Le pronom anaphorique de cette classe est yí.

\section{Les mots soumis à l'accord de classe}

Les mots qui s'accordent en minyanka sont : les pronoms anaphoriques (pronoms de classe), les pronoms interrogatifs, le pronom réfléchi, le pronom d'appartenance (pronom possessif), le pronom d'indéfini (pronom individualisateur), le démonstratif, le pronom 'l'autre', le déterminant 'autre', le relativiseur, les adjectifs, les marqueurs du présentatif et de l'identification.

\subsection{Les pronoms}

On peut classer les pronoms en allocutifs (voir Tableau 16) et en anaphoriques ou pronoms de classe (voir Tableau 17). Chacun de ces 
deux types se subdivisent en pronoms non-emphatiques et en pronoms emphatiques. Les allocutifs sont les pronoms de $1^{\text {ère }}$ et de $2^{\text {ème }}$ personne. Les pronoms non-emphatiques se distinguent des emphatiques par le fait qu'ils ne peuvent pas être utilisés seuls comme réponse à une question partielle.

Tableau 16

Pronoms allocutifs

\begin{tabular}{|l|l|l|}
\hline personne & non-emphatiques & emphatiques \\
\hline 1SG & ná & mè \\
\hline 2SG & má & mú \\
\hline $1 \mathrm{PL}$ & wò & wòrò \\
\hline 2PL & $y \grave{~}$ & yèrè \\
\hline
\end{tabular}

Tableau 17

Pronoms anaphoriques

\begin{tabular}{|l|l|l|}
\hline classe & non-emphatiques & emphatiques \\
\hline $\mathrm{W}$ & wú & wéré \\
\hline $\mathrm{P}$ & $p i ́$ & péré \\
\hline $\mathrm{K}$ & $k i ́$ & kéré \\
\hline $\mathrm{Y}$ & $y i ́$ & yéré \\
\hline $\mathrm{L}$ & lí & léré \\
\hline $\mathrm{T}$ & tí & téré \\
\hline
\end{tabular}

\subsection{Le pronom réfléchi}

Le pronom réfléchi est constitué d'un pronom non emphatique et du suffixe $-y \mathcal{\varepsilon}$ (probablement issu du déterminant $y \dot{\varepsilon}$ 'seul'). La forme réfléchie des pronoms allocutifs et celle des pronoms de classe sont données respectivement dans les Tableaux 18 et 19 . 
Tableau 18

Forme réfléchie des pronoms allocutifs

\begin{tabular}{|c|c|}
\hline personne & pronom réfléchi \\
\hline $1 \mathrm{SG}$ & náyغ̀ \\
\hline $2 \mathrm{SG}$ & máyè \\
\hline $1 \mathrm{PL}$ & wòyغ̀ \\
\hline $2 \mathrm{PL}$ & yèyè \\
\hline
\end{tabular}

Tableau 19

Forme réfléchie des pronoms de classe

\begin{tabular}{|c|c|}
\hline classe & pronom réfléchi \\
\hline $\mathrm{W}$ & wúyè \\
\hline $\mathrm{P}$ & píyè \\
\hline $\mathrm{K}$ & kíyè \\
\hline $\mathrm{Y}$ & yíỳ̀ \\
\hline $\mathrm{L}$ & líyè \\
\hline $\mathrm{T}$ & tíyè \\
\hline
\end{tabular}

\subsection{L'interrogatif}

Le déterminant interrogatif du minyanka est kè 'quel(les)' ; il se postpose au nom qu'il détermine et ne s'accorde pas (8b)-(9b). Cependant, le pronom interrogatif s'accorde avec le nom qu'il représente. Il est constitué d'un pronom non-emphatique auquel s'adjoint le déterminant interrogatif $(8 c)-(9 c)$.

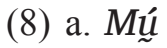
yá
cวั
nà lá ?
2SG.EMPH
PFV.AFF
femme.SG.DEF
voir Q
'As-tu vu la femme?'
b.
Cô
$k \grave{\varepsilon}$ ?
femme.sG.DEF quel
'Quelle femme?'


c. Wú-kì ?

3sG.w-quel

'Laquelle (femme) ?'

(9)

$\begin{array}{llll}\text { a. } \begin{array}{c}\text { - } b \grave{l} l \grave{c} \\ \text { femme-PL.INDF }\end{array} & \text { pá } & \text { pà } & \text { lá ? } \\ & \text { venir } & \text { Q }\end{array}$

'Les femmes sont-elles venues ?'

b. Cì-bèlè $\quad k \hat{\varepsilon}$ ?

femme-PL.INDF quel

'Quelles femmes ?'

c. $P \hat{i}-k \hat{\varepsilon}$ ?

3PL.P-quel

'Lesquelles (femmes) ?'

La liste des pronoms interrogatifs est donnée dans le Tableau 20.

Tableau 20

Pronoms interrogatifs

\begin{tabular}{|c|c|}
\hline classe & pronom interrogatif \\
\hline $\mathrm{W}$ & wúkè \\
\hline $\mathrm{P}$ & píks̀ \\
\hline $\mathrm{K}$ & $k i ̂ k \grave{\varepsilon}$ \\
\hline $\mathrm{Y}$ & yîkè \\
\hline $\mathrm{L}$ & lîkغ̀ \\
\hline $\mathrm{T}$ & tíkè \\
\hline
\end{tabular}

\subsection{Le pronom d'appartenance (pronom possessif)}

Le relateur possessif du minyanka de Pénesso est mó et ne s'accorde pas. Par contre, le pronom d'appartenance s'accorde avec la classe du nom qu'il représente. Il est constitué du relateur possessif auquel s'adjoint un suffixe nominal de classe, qui peut être celui du défini ou celui de l'indéfini. Le pronom d'appartenance doit être précédé d'un 
constituant nominal qui assume la fonction de dépendant. Les exemples suivants permettent de montrer que le pronom d'appartenance peut être utilisé à la forme indéfinie et à la forme définie. En (10b) et (11b), le pronom d'appartenance est à la forme indéfinie ; alors qu'en (10d) et (11d), il est à la forme définie.

(10) a. mè

mó $\quad c \dot{\varepsilon}-\varnothing$

1sG.EMPH POSS femme-SG.INDF

'une de mes femmes'

b. mè mó- $\emptyset$

1SG.EMPH POSS-W

'(litt.) Une (femme) m'appartenant'

c. mè mó cô

1SG.EMPH POSS femme.SG.DEF

'ma femme'

d. mè mô

1sG.EMPH POSS.W

'la mienne (femme)'

(11) a. Bákárì mó cí-ké

Bakary POss arbre-SG.INDF

'un arbre appartenant à Bakary (prénom masculin)'

b. Bákárì mó-kó

Bakary POSS-K

'(litt.) un (arbre) de Bakary'

c. Bákárì mó cí-kí

Bakary POSS arbre-SG.DEF

'l'arbre de Bakary'

d. Bákárì mó-kì

Bakary POSS-K

'celui de Bakary'

Les formes du pronom d'appartenance sont données dans le Tableau 21. 
Tableau 21

Formes du pronom d'appartenance

\begin{tabular}{|l|l|l|}
\hline classe & formes de l'indéfini & formes du défini \\
\hline $\mathrm{W}$ & mó & mô \\
\hline $\mathrm{P}$ & móolò & móolòpí \\
\hline $\mathrm{K}$ & mókó & mókì \\
\hline $\mathrm{Y}$ & móyó & móyì \\
\hline $\mathrm{L}$ & móló & mólì \\
\hline $\mathrm{T}$ & móró & mórì $\sim$ móorì \\
\hline
\end{tabular}

\subsection{Le pronom indéfini $C$-à}

Le pronom indéfini $C$-à 'un certain', où $C$ désigne la marque d'accord de classe, peut être utilisé en fonction de déterminant pour marquer les noms dont le référent est spécifique mais non identifiable par l'énonciateur. Ce pronom est appelé 'pronom individualisateur' par Manessy (1996a : 57). Les formes du pronom indéfini sont présentées dans le Tableau 22.

\section{Tableau 22}

Pronom indéfini selon la classe du nom-tête

\begin{tabular}{|l|l|}
\hline classe & pronom indéfini \\
\hline $\mathrm{W}$ & wà \\
\hline $\mathrm{P}$ & pà $\sim$ pèlè \\
\hline $\mathrm{K}$ & kà \\
\hline $\mathrm{Y}$ & $y \grave{a}$ \\
\hline $\mathrm{L}$ & là \\
\hline $\mathrm{T}$ & tà \\
\hline
\end{tabular}

\subsection{Le démonstratif}

Le démonstratif du minyanka est constitué de la forme nasalisée de la consonne de classe et du morphème -é. Les consonnes de classe non- 
obstruantes $w, l$ et $y$ se nasalisent en gardant leur lieu d'articulation, et les consonnes occlusives non-voisées $p, k$ et $t$ deviennent prénasalisées voisées. Les formes du démonstratif sont présentées dans le Tableau 23.

Tableau 23

Les formes du démonstratif selon la classe du nom-tête

\begin{tabular}{|l|l|}
\hline classe & démonstratif \\
\hline $\mathrm{W}$ & gé \\
\hline $\mathrm{P}$ & mbé $\sim$ mbélé \\
\hline $\mathrm{K}$ & $\eta g e ́$ \\
\hline $\mathrm{Y}$ & né \\
\hline $\mathrm{L}$ & né \\
\hline $\mathrm{T}$ & ndé \\
\hline
\end{tabular}

En minyanka, dans un syntagme DEM $+\mathrm{N}$, le ton final de $\mathrm{N}$ devient bas s'il était haut.
a. ฤé fyô
DEM.W poisson.SG.DEF
'ce poisson.' (cf. fyó)
DEM.K arbre-SG.DEF
'cet arbre' (cf. cíkí)
c. jgé yó-ৎóyì
DEM.K eau-SG.DEF
'cette eau' (cf. yóৎóyí)

b. jgé cí-kì

\subsection{Le pronom 'I'autre'}

Pour dire 'l'autre', comme dans l'énoncé : 'pas celui-ci, mais l'autre', le minyanka utilise un pronom composé que l'on peut analyser comme l'association d'un démonstratif et d'un pronom anaphorique. Lorsqu'il est utilisé comme déterminant, il a la propriété de pouvoir être antéposé 
ou postposé au nom qu'il détermine. Lorsqu'il est antéposé à un nom à schème tonal $\mathrm{H}$, le dernier ton de celui-ci devient $\mathrm{B}$ (13a). Mais lorsqu'il lui est postposé, le nom déterminé ne subit aucun changement tonal (13b). En (13c), il est utilisé de façon pronominale.
(13) a. நgé-kì
$c i ́-k i ̀$
DEM.K-3SG.K arbre-SG.DEF
'l'autre arbre'
b. $\quad c i-k i$
jgé-ki
arbre-SG.DEF DEM.K-3SG.K
'l'autre arbre' (même sens que (13a))
c. Jgé-kì wá tò.
DEM.K-3SG.K PRF.AFF tomber
'L'autre (arbre) est tombé.'

Les différentes formes de 'l'autre' sont présentées dans le Tableau 24.

Tableau 24

Les formes du pronom 'l'autre' selon la classe du nom-tête

\begin{tabular}{|l|l|}
\hline classe & 'l'autre, les autres' \\
\hline $\mathrm{W}$ & jéwù \\
\hline $\mathrm{P}$ & mbélépì \\
\hline $\mathrm{K}$ & ggékì \\
\hline $\mathrm{Y}$ & néyì \\
\hline $\mathrm{L}$ & nélì \\
\hline $\mathrm{T}$ & ndérì \\
\hline
\end{tabular}

Comme nous pouvons le constater, tous les pronoms anaphoriques suffixés au démonstratif portent un ton bas ; cela est dû au fait que les démonstratifs sont suivis d'un ton flottant bas. Dans la forme du pronom ndé-rì, il y a une alternance $t / r$; la consonne initiale du pronom tí est remplacée par $r$. 


\subsection{Le déterminant 'autre'}

Le minyanka de Pénesso dispose de deux mots : $C$-àbè' et $C$-àtè que l'on peut traduire par 'autre' bien qu'ils ne soient pas toujours interchangeables. Le mot $C$-àbè' (suivi d'un ton flottant haut) est étymologiquement constitué du pronom indéfini $C$-à et de bé 'aussi'. On l'utilise uniquement pour désigner une chose qui se rajoute à une autre de même nature. Quant à $C$-àtè, il est généralement utilisé pour désigner une chose différente de la première. Comparer (14a-b) et (15a-b). L'énoncé (14b) est généralement compris comme la femme qu'il avait auparavant est partie.
(14) a. Wú
wá
cว̆
w-àbè ló.
3SG.W PRF.AFF femme.SG.DEF W-autre prendre
'Il a pris une nouvelle femme (en gardant la première).'
b. Wú wá cว̌ w-àtè lò.
3SG.W PRF.AFF femme.SG.DEF W-autre prendre
'Il a pris une autre femme.'

En (15a), on ne peut pas substituer $w$-àbè à $w$-àtè d'où l'agrammaticalité de (15b).
(15) a. $C \dot{\varepsilon}-\varnothing$
femme-SG.INDF
gé-mì bè
wù
$m a ́$
wú yá w-èrè yácà má w-àtè lò.
3SG.W PFV.AFF W-EMPH laisser et.ss w-autre prendre
'Il a répudié la femme qu'il avait et en a épousé une autre.'
b.

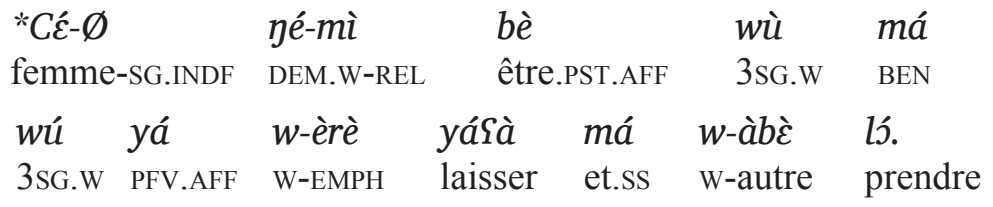

En (16)-(17) C-àbè et C-àtè sont utilisés de façon pronominale, mais seul $C$-àtè peut figurer dans une phrase négative (17b). 
(16) a. W-àbè wá pà.

W-autre PRF.AFF venir

'Une autre personne est venue (s'ajouter à une ou d'autres personnes déjà présentes).'

b. *W-àbc̀ wâ pà méc.

W-autre PRF.NEG venir NEG

(sens recherché) 'Personne d'autre n'est venu.'

(17) a. W-àtè yà pà.

W-autre PFV.AFF venir

'Une tout autre personne est venue (une personne à qui on ne s'attendait pas).'

b. W-àtè kă pà méc.

W-autre PFV.NEG venir NEG

'Personne d'autre n'est venu.'

Les différentes formes de ces deux déterminants sont données dans le Tableau 25.

Tableau 25

Les formes de 'autre' selon les classes

\begin{tabular}{|c|c|c|}
\hline classe & autre $_{1}$ & autre $_{2}$ \\
\hline W & wàbè & wàtè \\
\hline $\mathrm{P}$ & pàbc̀ & pàtè \\
\hline K & kàbè & kàtè \\
\hline$Y$ & yàbc̀ & yàtè \\
\hline $\mathrm{L}$ & làbc̀ & làtè \\
\hline $\mathrm{T}$ & tàbc̀ & tàtè \\
\hline
\end{tabular}

\subsection{Le relativiseur}

La proposition relative n'est pas enchâssée en minyanka ; elle se place généralement à gauche de la proposition matrice. Elle est marquée par un relativiseur qui se place immédiatement après le nom relativisé. 
Le relativiseur a deux formes : mî et DEM-mì. La première ne s'accorde pas avec le groupe nominal relativisé, alors que la seconde, constituée d'un démonstratif et de mî dont le ton devient bas à cause du ton flottant bas suivant le démonstratif, s'accorde avec l'antécédent. Le mot mî est emprunté à la langue bambara. Ces deux formes du relativiseur sont interchangeables dans les cas où l'antécédent est exprimé (18a-b), mais seule la forme DEM-mì est admise dans les relatives libres (19a).
a. Má yá
2SG PFV.AFF

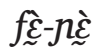
né-mì
jó
habit-PL.INDF
DEM.Y-REL acheter
$\begin{array}{llll}\text { tánâaa, } & y i ́ & w a ́ & c \grave{r} r \dot{\varepsilon} . \\ \text { hier } & \text { 3PL.Y } & \text { PRF.AFF } & \text { être.petit }\end{array}$

'Les habits que tu as achetés hier sont petits.'

$\begin{array}{llllll}\text { b. } & \text { Má } & y a ́ & f \hat{\varepsilon}-n \grave{\varepsilon} & m \hat{\imath} & \int \mathfrak{\jmath} \\ & 2 \mathrm{SG} & \text { PFV.AFF } & \text { habit-PL.INDF } & \text { REL } & \text { acheter }\end{array}$

tánâa, yí wá cèré.

hier 3PL.Y PRF.AFF être.petit

'Les habits que tu as achetés hier sont petits.'

(même sens que (18a))

(19)
a. Má yá jè-mî fó tánẫa,
2SG PFV.AFF DEM.Y-REL acheter hier
yí wá cèré
3PL.Y PRF.AFF être.petit
'Ceux (les habits) que tu as achetés hier sont petits.'
b. *Má yá mî fó tánâaa, yí 2SG PFV.AFF REL acheter hier 3PL.Y
wá cèré
PRF.AFF être.petit

Les différentes formes du relativiseur sont données dans le Tableau 26. 
Tableau 26

Les différentes formes du relativiseur selon les classes

\begin{tabular}{|l|l|}
\hline classe & relativiseur \\
\hline $\mathrm{W}$ & gémì \\
\hline $\mathrm{P}$ & mbélémì \\
\hline $\mathrm{K}$ & ggémi \\
\hline $\mathrm{Y}$ & némì \\
\hline $\mathrm{L}$ & némì \\
\hline $\mathrm{T}$ & ndémì \\
\hline
\end{tabular}

\subsection{Les adjectifs}

Il existe deux types d'adjectifs en minyanka : les adjectifs non autonomes et les adjectifs autonomes. Les premiers sont des mots qui ne peuvent pas apparaître seuls et s'incorporent entre la base et le suffixe nominal de classe du nom qu'ils qualifient. Ils sont une dizaine en minyanka : -fu- 'chaud', -fi- 'blanc', - $л \varepsilon$ - 'rouge', -pó- 'gros (morceau de)', -go-

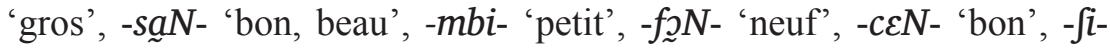
'premier'. Les seconds, qui sont étudiés ici, sont formés à partir du préfixe adjectival wóN- qui s'adjoint à des adjectifs non autonomes pour donner des adjectifs autonomes et à des verbes pour donner des participes qui fonctionnent comme adjectifs. La nasale sous-jacente de ce préfixe modifie la consonne initiale du mot auquel ce préfixe s'adjoint. Lorsque la consonne initiale de ce mot est une non-obstruante $(l, y, w)$, celle-ci se nasalise en gardant son lieu d'articulation. Lorsqu'elle est une fricative non-voisée, celle-ci devient voisée. Lorsqu'elle est une occlusive nonvoisée, celle-ci se transforme en occlusive voisée prénasalisée. En revanche, lorsque le mot auquel wóN-s'adjoint commence par une consonne nasale, celle-ci ne subit aucune modification.

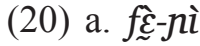
$w \grave{o}-n \hat{\varepsilon}-y \grave{~}$
habit-PL.DEF
ADJ-être.vieuX-Y
'les vieux habits' (lı̀ 'être vieux') 


\section{b. fề-nì wò-ngóló-nì. \\ habit-PL.DEF ADJ-être.laid-Y}

'Les mauvais habits.' (kòlô 'être laid')
c. Jú-rì wò-vú-rì
tô-DEF ADJ-chaud-T
'Du tô (type de plat) chaud.' (-fu- 'chaud')

d. fर्षे-nì wò-nर́-yì

habit-PL.DEF ADJ-rouge-Y

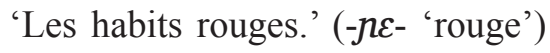

\subsection{Le déterminant $s a ̊ \sim N-C L$}

Le déterminant sá $N$-CL signifie 'le reste de, restant' comme dans : 'Sur 15 élèves, seuls 5 sont venus, le reste (les autres) était absent'. La nasale sous-jacente de $s a \sim \alpha N$ assimile la consonne du suffixe nominal de classe. Lorsque sá $N$-CL détermine un nom appartenant à la classe $\mathrm{W}$, il a la même forme que lorsqu'il détermine un nom de la classe $\mathrm{K}$. Ce déterminant peut être utilisé à la forme indéfinie et à la forme définie. Les formes de l'indéfini et du défini de ce déterminant sont présentées dans le Tableau 27.

Tableau 27

Formes du déterminant sá $N$-CL selon les classes

\begin{tabular}{|l|l|l|}
\hline classe & formes de l'indéfini & formes du défini \\
\hline $\mathrm{W}$ & sánáa & sánì \\
\hline $\mathrm{P}$ & sàmànà & sàmànàpì \\
\hline $\mathrm{K}$ & sánáa & sánì \\
\hline $\mathrm{Y}$ & sánáa & sánì \\
\hline $\mathrm{L}$ & sǻná & sánì \\
\hline $\mathrm{T}$ & sắrá & sárì \\
\hline
\end{tabular}

\subsection{Le marqueur d'identification}

Le marqueur d'identification est $C$-í pour la forme non emphatique et $C-\hat{\varepsilon}$ pour la forme emphatique. La forme non emphatique se postpose 
immédiatement au constituant nominal en position de sujet et s'accorde en classe (21a-b). La forme emphatique s'accorde aussi en classe et est reliée au sujet par la copule wá 'être.PRS' ou bé 'être.PST' (22a-c), et sert à marquer l'insistance.
a. Yó-Sóyí $k$-í.
eau-SG.DEF K-ID
'C'est de l'eau.'

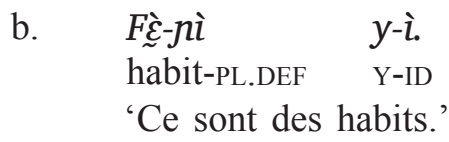

(22)
a. Yó-ৎóyí
wá
$k-\hat{\varepsilon}$.
eau-SG.DEF être.PRS.AFF K-ID.EMPH

'C'est de l'eau (et non autre chose).'

b.

F⿳亠े⿵-nì wà $\quad y$-ह̂.

habit-PL.DEF être.PRS.AFF Y-ID.EMPH

'Ce sont des habits (et non autre chose).'
c. F⿳亠丷⿵冂- $n \grave{i}$ bè $y-\hat{\varepsilon}$.
habit-PL.DEF être.PST.AFF Y-ID.EMPH
'C'étaient des habits.'

\subsection{Le marqueur du présentatif}

Le marqueur du présentatif a aussi 2 formes : C-é pour la forme non emphatique et $C$-érè pour la forme emphatique. Elles se placent immédiatement après le constituant nominal en position de sujet, et s'accordent en classe. La forme non emphatique du marqueur du présentatif prend le dernier ton du nom qui la précède (23a) et (24a). Le schème tonal de la forme emphatique est $\mathrm{H}$-B lorsque le nom en position de sujet se termine par un ton haut, et BH-B lorsqu'il se termine par un ton bas (23b) et (24b).
a. Yó-Yóyí $k$-é.
eau-SG.DEF K-PRSTF
'Voici de l'eau.' 

b. Yó-ৎóyí k-é-rè.
eau-SG.DEF K-PRSTF-EMPH
'Voici de l'eau (avec insistance)'.

(24)
a. Háa-rì
$t-\grave{e}$.
viande-DEF
T-PRSTF

'Voici de la viande.'

\section{b. Háa-rì t-ě-rè. \\ viande-DEF T-PRSTF-EMPH}

'Voici de la viande (avec insistance).'

L'ensemble des mots soumis à l'accord de classe sont repris dans le Tableau 28. Voici la liste des abréviations qui sont utilisées dans ce tableau : APPART - pronom d'appartenance (pronom possessif), DEM démonstratif, EMPH - emphatique, ID - marqueur d'identification, INDF - indéfini, INTER - interrogatif, PR - pronom, PRSTF marqueur du présentatif, REFL - réfléchi, REL - relativiseur.

Tableau 28

Les mots soumis à l'accord de classe

\begin{tabular}{|c|c|c|c|c|c|c|}
\hline & $\mathbf{W}$ & $\mathbf{P}$ & $\mathbf{K}$ & $\mathbf{Y}$ & $\mathbf{L}$ & $\mathbf{T}$ \\
\hline PR non EMPH & wú & pí & kí & yí & lí & tí \\
\hline PR EMPH & wéré & péré & kéré & yéré & léré & téré \\
\hline PR REFL & wúyè & píyè & kíyè & yíyè & líyદ̀ & tíyè \\
\hline PR INTER & $w u ́ k \grave{\varepsilon}$ & píkè & kíkè & yíkè & líkغ̀ & tíkè \\
\hline DEM & yé & $\begin{array}{l}\text { mbélé 〜 } \\
\text { mbé }\end{array}$ & ggé & jé & né & ndé \\
\hline REL & yé-mì & $\begin{array}{l}\text { mbélémì } \\
\text { mbémì }\end{array}$ & ggémì & némì & némì & ndémì \\
\hline PR INDF & $w a ̀$ & pà pèlè & $k a ̀$ & yà & là & tà \\
\hline 'autre, & 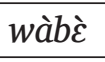 & pàbè & $k a ̀ b \varepsilon ̀ ~$ & yàbc̀ & làbè & tàbè \\
\hline 'autre, & wàtè & pàtè & kàtè & yàtè & làtè & tàtè \\
\hline 'l'autre' & géwù & mbélépì & ggékì & néyì & nélì & ndérì \\
\hline
\end{tabular}


Fin du tableau 28

\begin{tabular}{|l|l|l|l|l|l|l|}
\hline & W & $\mathbf{P}$ & $\mathbf{K}$ & $\mathbf{Y}$ & $\mathbf{L}$ & $\mathbf{T}$ \\
\hline restant & sánì & sàmànàpì & sánì & sánì & sánì & sárì \\
\hline APPART & mô & móolòpí & mókì & móyì & mólì & mórì \\
\hline ID & $w i ́$ & $p i ́$ & $k i ́$ & $y i ́$ & $l i ́$ & tí \\
\hline ID EMPH & $w \hat{\varepsilon}$ & $p \hat{\varepsilon}$ & $k \hat{\varepsilon}$ & $y \hat{\varepsilon}$ & $l \hat{\varepsilon}$ & $t \hat{\varepsilon}$ \\
\hline PRSTF & $w e ́$ & $p e ́$ & $k e ́$ & $y e ́$ & $l e ́$ & té \\
\hline PRSTF EMPH & wérè & pérè & kérè & yérè & lérè & térè \\
\hline
\end{tabular}

\section{Conclusion}

Si nous comparons le minyanka de Pénesso aux autres parlers minyanka déjà décrits cf. Cauvin (1980), Dombrowsky-Hahn (1999; 2007), nous nous rendons compte que ce parler a subi une réduction du nombre de ses classes d'accord. En effet, dans les autres parlers minyanka déjà décrits, on distingue clairement 8 suffixes nominaux de classe et 8 classes d'accord, comme nous pouvons le constater dans la Figure 3 empruntée à Dombrowsky-Hahn (2007: 332) sur le parler de Yorosso. Cette figure présente les suffixes de l'indéfini des noms et les pronoms anaphoriques (en caractère gras) de chaque classe.

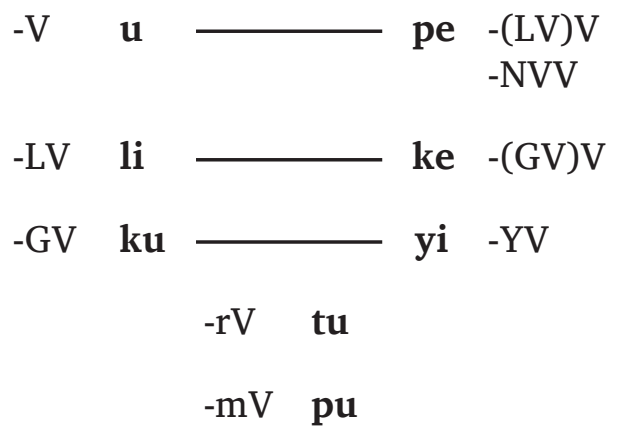

Figure 3. Suffixes de l'indéfini et pronoms de classe du minyanka d'après Dombrowsky-Hahn (2007: 332) 
On constate dans la Figure 3 que chaque forme nominale appartient à une classe d'accord différente, symbolisée par la forme du pronom anaphorique. Or si nous nous reportons à la figure 1 sur le minyanka de Pénesso, nous constatons que les noms à suffixe $-K V l V$ (pluriel des noms à suffixe $-L V$ ) et les noms à suffixe $-r V$ (pluriel de certains noms à suffixe $-L V$, et suffixe des noms collectifs) appartiennent à la même classe d'accord : la classe $\mathrm{T}$; les noms à suffixe de singulier $-K V$ et les noms à suffixe $-m V$ (classe des liquides) s'accordent en classe $\mathrm{K}$; le nom jómí 'les paroles', qui a une valeur de pluriel en minyanka et les noms à suffixe de pluriel $-Y V$ s'accordent en classe Y. En d'autres termes, dans le parler de Pénesso, les noms au pluriel désignant les petites choses et les noms collectifs sont mis ensemble dans la même classe d'accord. Les noms désignant les non-humains (noms à suffixe $-K V$ ) et les noms désignant les liquides (noms à suffixe $-m V$ ) sont mis ensemble dans la même classe d'accord : la classe K. Comme le nom jómí 'les paroles' a une valeur de pluriel, il s'accorde en classe Y, comme le pluriel des noms à suffixe $-K V$.

Quand on compare les différentes langues sénoufo, on se rend compte que des cas de réduction de classes sont attestés dans certaines d'elles, telles que le tafire et le dyimini, qui ont 7 classes, et le kar, qui en a 6, cf. les configurations de Manessy (1996b : 24). Même si le kar a aussi 6 classes d'accord d'après Manessy, son système de classes n'a pas la même configuration que celui de Pénesso. Le kar a un pronom de structure $t V$ comme pluriel du pronom de structure $k V$, et un pronom $k V$ comme pluriel du pronom de structure $l V$. Or en minyanka de Pénesso, le pronom tí est plutôt le pluriel du pronom lí, et le pronom yí est le pluriel du pronom kí.

La configuration des classes d'accord dans le minyanka de Pénesso constitue à ce jour un cas unique parmi les langues sénoufo déjà décrites. La description des autres langues sénoufo permettra de dire si d'autres langues de ce groupe ont ou non la même configuration que le minyanka de Pénesso. 


\section{Abréviations}

ADJ - préfixe adjectival
AFF - affirmatif
BEN - postposition à valeur bénéfactive
CL - marqueur de classe
DEF - défini
DEM - démonstratif
EMPH - emphatique
ID - marqueur d'identification
INDF - indéfini
NEG - marque de négation
PFV - perfectif (accompli)

$\mathrm{PL}$ - pluriel

POSS - possessif

PRF - parfait

PRS - présent

PRSTF - marqueur du présentatif

PST - passé

$\mathrm{Q}$ - particule interrogative

REL - relativiseur

$\mathrm{SG}-$ singulier

ss - même suject

\section{Références}

Carlson, Robert. 1994. A grammar of Supyire. Berlin: Mouton de Gruyter.

Cauvin, Jean. 1980. L'image, la langue et la pensée. Saint Augustin: Anthropos-Institut Haus Völker u. Kulturen.

Dombrowsky-Hahn, Klaudia. 1999. Phénomènes de contact entre les langues minyanka et bambara (Sud du Mali). Köln: Rüdiger Köppe.

Dombrowsky-Hahn, Klaudia. 2007. Minyanka. In Miehe, Gudrun \& Winkelmann, Kerstin (eds.), Noun class systems in Gur languages, 331-354. Cologne: Rüdiger Köppe. (Gur Monographs 9)

Eberhard, David M. \& Simons, Gary F. \& Fennig, Charles D. (eds.). 2021. Ethnologue: Languages of the world. $24^{\text {th }}$ edition. Dallas, Texas: SIL International. Online version: http://www.ethnologue.com

Güldemann, Tom \& Fiedler, Ines. 2019. Niger-Congo "noun classes" conflate gender with deriflection. In Di Garbo, Francesca \& Olsson, Bruno \& Wälchli, Bernhard (eds.), Grammatical gender and linguistic complexity. Vol 1. General issues and specific studies, 95-145. Berlin: Language Science Press.

Manessy, Gabriel. 1996a. La détermination nominale en sénoufo. Linguistique Africaine 16, 53-68. 
Manessy, Gabriel. 1996b. Observations sur la classification nominale en sénoufo. Afrika und Übersee 79(1), 21-35.

Miehe, Gudrun \& Winkelmann, Kerstin (eds.). 2007. Noun class systems in Gur languages. Cologne: Rüdiger Köppe. (Gur Monographs 9.) 